\section{THE ASSOCIATION BETWEEN WORLD DEVELOPMENT INDICATORS PER COUNTRY AND GENDER RATIO IN PATIENTS WITH SYSTEMIC LUPUS ERYTHEMATOSUS: A BIG DATA APPROACH ANALYSIS}

${ }^{1} \mathrm{M}$ Pérez de Lis Novo, ${ }^{2} R$ Pérez-Álvarez ${ }^{*},{ }^{3} \mathrm{~B}$ Kostov, ${ }^{4} \mathrm{P}$ Brito-Zerón, ${ }^{5} \mathrm{M}$ Gandía, ${ }^{6} \mathrm{~A}$ SisoAlmirall, ${ }^{7} \mathrm{D}$ Superville, ${ }^{8} \mathrm{Y}$ Shoenfeld, ${ }^{9} \mathrm{M}$ Ramos-Casals, ${ }^{10} \mathrm{MA}$ Khamashta. ${ }^{1}$ Hospital Juan Canalejo, Anesthesiology and Intensive Care, A Coruña, Spain; ${ }^{2}$ Hospital Alvaro Cunqueiro, Internal Medicine- Department of Autoimmune Diseases., Vigo, Spain; ${ }^{3}$ Primary Care Centre Les Corts- CAPSE, Institut d'Investigacions Biomèdiques August Pi i Sunyer IDIBAPS, Barcelona, Spain; ${ }^{4}$ Josep Font Laboratory of Autoimmune Diseases- CELLEX-Institut d'Investigacions Biomèdiques August Pi i Sunyer IDIBAPS, Department of Autoimmune Diseases- ICMiD-Hospital Clínic, Barcelona, Spain; ${ }^{5}$ Hospital Puerta del Mar, Rheumatology, Cádiz, Spain; ${ }^{6}$ Primary Care Research Group- Institut d'Investigacions Biomèdiques August Pi $i$ Sunyer IDIBAPS, Primary Care Centre Les Corts- CAPSE, Barcelona, Spain; ${ }^{7}$ Massachusetts Institute of Technology, MIT, Cambridge- Massachusetts, USA; ${ }^{8}$ 7Zabludowicz Centre for Autoimmune Diseases- Chaim Sheba Medical Center- Tel Hashomer-Israel, Incumbent of the Laura Schwarz-Kipp Chair for Research of Autoimmune Diseases- Sackler Faculty of Medicine- Tel-Aviv University, Tel-Aviv, Israel; ${ }^{9}$ osep Font Laboratory of Autoimmune Diseases- CELLEX-Institut d'Investigacions Biomèdiques August Pi i Sunyer IDIBAPS-, Department of Autoimmune Diseases- ICMiD- Hospital Clinic, Barcelona, Spain; ${ }^{10}$ St Thomas' Hospital- King's College University, Lupus Research UnitThe Rayne Institute, London, UK

\subsection{6/lupus-2017-000215.415}

Background and aims Systemic autoimmune diseases (SAD) are characterised by a wide spectrum of demographic patterns with respect to the ethnic differences, age at diagnosis and especially gender distribution. Studying the distribution of these diseases across geographic regions using a big datadriven approach may help obtain a more "high-definition resolution" of these complex diseases.

Methods We explored the potential of the Google search engine to collect and merge 133 SLE cohorts (>100 patients) reported in the Pubmed library. The country indicators are subclassified into 20 specific topics.Statistically-significant correlations were further corrected according to the Lasso statistical model (LC).

Results We found statistical correlations in the following areas: Education, Environment, Infrastructure, Economy and Growth, Health, Private sector, Public sector and Social Protection and Labour. A higher F:M ratio was found in countries who had a higher frequency of women in tertiary education/academic staff ,female legislators, higher\% of CO2 emissions from electricity/heat, higher $\%$ of terrestrial and marine protected areas and of taxes. In contrast, a lower F:M ratio was found in countries who had a higher frequency of women in unemployment and countries with a higher out-of-pocket health expenditure for private healthcare

Conclusions There is a clear trend of association between the percentage of women diagnosed with SLE and some indicators of development of each country. The gap between women and men diagnosed with SLE is wider in countries with the highest frequencies of women working and women with high study degrees, and those countries with more taxes and a higher percentage of protected geographical areas.

\section{BIG DATA IN SYSTEMIC LUPUS ERYTHEMATOSUS: PHENOTYPIC DISEASE EXPRESSION OF 171,000 ADULT PATIENTS}

${ }^{1} \mathrm{M}$ Pérez de Lis Novo, ${ }^{2} \mathrm{M}$ Gandía, ${ }^{3} \mathrm{R}$ Pérez- Álvarez ${ }^{*}$, ${ }^{4}$ Brito-Zerón, ${ }^{5} \mathrm{~B}$ Kostov, ${ }^{5} \mathrm{~A}$ SisoAlmirall, ${ }^{6} \mathrm{D}$ Superville, ${ }^{7} Y$ Shoenfeld, ${ }^{4} \mathrm{M}$ Ramos-Casals, ${ }^{8} \mathrm{MA}$ Khamashta. ${ }^{1}$ Hospital Juan Canalejo, Anesthesiology and Intensive Care, A Coruña, Spain; ${ }^{2}$ Hospital del Mar, Rheeumatology, Cadiz, Spain; ${ }^{3}$ Hospital Alvaro Cunqueiro, Department of Autoimmune Diseases, Vigo, Spain; ${ }^{4}$ Josep Font Laboratory of Autoimmune Diseases- CELLEX-Institut d'Investigacions Biomèdiques August Pi i Sunyer IDIBAPS, Department of Autoimmune Diseases- ICMiD- Hospital Clínic, Barcelona, Spain; ${ }^{5}$ Primary Care Research Group- Institut d'Investigacions Biomèdiques August Pi i Sunyer IDIBAPS, Primary Care Centre Les CortsCAPSE, Barcelona, Spain; ${ }^{6}$ Massachusetts Institute of Technology, MIT, CambridgeMassachusetts, Spain; 'Zabludowicz Centre for Autoimmune Diseases- Chaim Sheba Medical Center- Tel Hashomer- Israel, Incumbent of the Laura Schwarz-Kipp Chair for Research of Autoimmune Diseases- Sackler Faculty of Medicine- Tel-Aviv University, TelAvivi, Spain; ${ }^{8}$ St Thomas' Hospital- King's College University, Lupus Research Unit- The Rayne Institute, London, Spain

\subsection{6/lupus-2017-000215.416}

Background and aims Studying the distribution of SLE across geographic regions using a big data-driven approach may facilitate understanding of the corresponding genetic and environmental underpinnings.

Methods We explored the potential of the Google search engine to collect and merge cohorts (>100 patients) of patients with systemic lupus erythematosus (SLE) reported in the Pubmed library. We made a text-word search in Google between 8th and 15th May 2015 using SLE and "100...100000000 patients" and "site:http://www.ncbi.nlm.nih. gov/pubmed". We collected the available data about study design, country, ethnicities, age and gender, clinical features and immunological markers.

Results We merged the data of 133 SLE cohorts including 171000 patients; gender was detailed in 130 cohorts: $88 \%$ women(female:male ratio, 8,4). mean age at onset $(29.89$ $\pm 3.48)$, at diagnosis $(32.33 \pm 2.99)$. The countries contributing the most cohorts were the USA (31), Japan (8) and Spain (5). The main clinical features included arthritis in $72 \%$, haematological abnormalities in 62\%, malar rash in 50\%,photosensitivity in $48 \%$, renal involvement in $38 \%$, oral ulcers in $34 \%$, serositis in $30 \%$ and neurological involvement in 14\%. Haematological abnormalities included lymphopenia in 43\%,leukopenia in 38\%,thrombocytopenia in $13 \%$ and hemolytic anaemia in 4\%.Positive autoantibodies included ANA in 91\%,dSDNA in $62 \%$,anti-Ro/SSA in $35 \%$,antiRNP in $25 \%$, antiSm in $21 \%$ and anti-La/SSB in $15 \%$.

Conclusions This is the largest reported study in SLE including nearly 200000 cases that provides a big data picture of the worldwide expression of the disease, with a female:male ratio of 8,4 , a mean age at diagnosis of 32 years, and with joints, haematological, skin and kidneys being the most frequent organs involved. 